\title{
State of Health Estimation Based on OS-ELM for Lithium-ion Batteries
}

Yiduo Zhu ${ }^{1,2,3}$, Fuwu Yan $^{1,2}$, Jianqiang Kang ${ }^{1,2}$,Changqing $\mathrm{Du}^{1,2, *}$

${ }^{1}$ Hubei Key Laboratory of Advanced Technology for Automotive Components, Wuhan University of Technology, Wuhan 430070, China

${ }^{2}$ Hubei Collaborative Innovation Center for Automotive Components Technology, Wuhan University of Technology, Wuhan 430070, China

${ }^{3}$ Wuhan Technical College of Communications, Wuhan 430065, China

*E-mail: cq_du@whut.edu.cn

doi: $10.20964 / 2017.07 .35$

Received: 5 April 2017 / Accepted: 29 April 2017 / Published: 12 June 2017

An accurate state of health ( $\mathrm{SOH}$ ) estimation can facilitate the design of reliable battery systems and ensure reliability and safety during battery operation. An effective prediction algorithm is indispensable in performing an accurate $\mathrm{SOH}$ estimation. In this study, to solve the problem of obtaining battery capacity fading under the real vehicle state, the discharge time of equal voltage interval is used as the health indicator of the battery. The selection reason of the discharge voltage interval is explained from the aspects of battery mechanism and experimental data. To solve the problem of accuracy and large computation in SOH estimation, an online sequential extreme learning machine is used to predict $\mathrm{SOH}$. The method demonstrates fast learning and generalization performance. The prediction error is less than $1.9 \%$, which proves the accuracy of the method.

Keywords: State of health, Extreme learning machine, Health indicator, Lithium-ion battery

\section{FULL TEXT}

(C) 2017 The Authors. Published by ESG (www.electrochemsci.org). This article is an open access article distributed under the terms and conditions of the Creative Commons Attribution license (http://creativecommons.org/licenses/by/4.0/). 